\title{
Efecto del preservado de la madera con boro sobre el módulo dinámico (evaluación por medio de ultrasonido)
}

\section{Javier Ramón Sotomayor-Castellanos}

Universidad Michoacana de San Nicolás de Hidalgo Facultad de Ingeniería en Tecnología de la Madera, Avenida Francisco J. Múgica $\mathrm{S} / \mathrm{N}$, Ciudad Universitaria, C.P. 58030, Morelia, Michoacán.

MEXICO

correo electrónico (email): madera999@yahoo.com

Recibido 03-09-2019, aceptado 21-11-2019.

\section{Resumen}

El objetivo de la presente investigación fue observar el efecto del tratamiento de preservación con sales de boro sobre la densidad, la velocidad de onda y el módulo de elasticidad dinámico en las maderas Guazuma ulmifolia, Spathodea campanulata y Abies religiosa. Se prepararon lotes de treinta y cinco probetas que se preservaron con el método baño caliente-frío, con una solución concentrada al tres por ciento. Para cada especie se determinó la densidad, la velocidad de onda y el módulo de elasticidad dinámico, antes y después del tratamiento. Además, se calculó la absorción y la retención de sales. Se realizaron pruebas de diferencia de medias y se calcularon regresiones para las variables, antes y después de tratamiento. Se encontró que el tratamiento de preservación modifica la densidad, la velocidad de ultrasonido y el módulo de elasticidad dinámico en las maderas G. ulmifolia, S. campanulata y A. religiosa. Sin embargo, la retención de sales de boro es una característica particular para la madera de cada especie. Este componente satisface los estándares necesarios para su empleo en condiciones donde se requiere un método de protección.

Palabras clave: absorción, baño caliente-frío, densidad de la madera, retención, velocidad de onda.

\section{Abstract \\ (Effect of wood preservation with boron on the dynamic modulus -evaluation by ultrasound)}

The goal of the research was to observe the effect of the preservation treatment with boron salts on the density, wave speed and dynamic modulus of elasticity in wood samples of Guazuma ulmifolia, Spathodea campanulata and Abies religiosa. Using the bath hot-cold method, thirty-five normalized specimens were prepared and preserved with a three percent concentration solution. For each species, density, wave velocity and dynamic modulus of elasticity were determined, before and after the treatment. In addition, absorption and salt retention were calculated. For the variables before and after the treatment, there were realized tests of difference of means, and were calculated models of regression. It was found that the impregnation treatment modifies the wood density, the wave speed and the dynamic modulus of elasticity of G. ulmifolia, S. campanulata and $A$. religiosa. However, the retention of boron salts for $G$. ulmifolia, $S$. campanulata and A. religiosa is particular to each species and satisfies the necessary standards for their use in conditions in which a protection treatment is required.

Index terms: absorption, bath hot-cold, retention, wave speed, wood density.

\section{Introducción}

La madera es una materia prima que compite con otros materiales y tecnologías propias en la industria de la construcción. Es recomendable el conocimiento fundamental de los atributos relativos a la aptitud de este material para construir, ello con el objeto de mejorar la productividad industrial. Uno de esos atributos es la capacidad para retener sustancias protectoras al ataque de agentes biológicos, como son los hongos y moho. Tales agentes producen un deterioro como resultado de exponer la madera a la humedad excesiva y por un largo periodo de tiempo. Los efectos de este proceso de deterioro se manifiestan cuando la superficie cambia de color, toma un aspecto sucio y muchas 
veces pierde brillo; así mismo, su cohesión de superficie disminuye y aumenta su fragilidad. Es entonces que la madera se fractura y se pueden desprender fragmentos de la misma [1].

No obstante que la madera es un material durable, la conservación de sus propiedades tecnológicas para su óptimo uso en la construcción depende del medio ambiente donde sea instalada. La madera sin protección expuesta al exterior sufre principalmente los efectos del fotodeterioro causado por la luz ultravioleta, la hidrólisis y la variación dimensional. Esto se produce debido a los cambios de humedad, además del biodeterioro y la decoloración derivada de la acción de microorganismos [2].

Cuando la madera no es tratada adecuadamente puede ser deteriorada por agentes biológicos y factores físicos, los cuales modifican sus elementos básicos provocando su gradual degradación. Por ejemplo, las enzimas, las substancias ácidas o la radiación electromagnética provocan reacciones químicas que modifican el estado de la superficie de la madera. Estas moléculas ya descompuestas perturban las partes de la anatomía celular, deteriorando los tejidos vegetales de la madera [3].

La tecnología de preservación de la madera por medio de arsénico, cobre y cromo [4] ha sido remplazada por el empleo de sustancias ecológicamente amigables [5]. Las sales trihidróxido de boro (ácido bórico) y tetraborato de sodio (borato de sodio), son utilizadas para proteger la madera del ataque de hongos e insectos [6]. Sus principales ventajas, respecto a otras substancias preservantes, es que son inodoras, incoloras e inflamables; además que poseen poca toxicidad para el ser humano [7]. El empleo de estas propiedades se ha generalizado para el caso de elementos estructurales en construcciones con madera. Sin embargo, las sales de boro se disuelven en contacto con agua, causando el proceso de lixiviado [8]. Por lo tanto, se recomienda su empleo en componentes que no están expuestos a la intemperie.

El módulo de elasticidad dinámico de la madera es el parámetro de ingeniería que se utiliza en el cálculo de estructuras construidas con este material [9]. Su determinación se realiza por medio de métodos normalizados en condiciones de cargas, estáticas o dinámicas, donde se emplean probetas de pequeñas dimensiones [10]. Así mismo, se utilizan otros métodos que miden la resistencia mecánica en elementos de madera con dimensiones de uso [11]. El empleo de métodos no destructivos en la determinación de propiedades mecánicas permite, igualmente, estudiar piezas de madera antes y después de ser expuestas a un tratamiento [12].
Las ondas acústicas pueden viajar a través de la madera. Se puede determinar el módulo de elasticidad dinámico [13] a partir del cálculo de su velocidad en conjunto con la densidad de la madera. Se puede medir la velocidad del ultrasonido en una especie, aplicar un tratamiento para mejorar alguna propiedad y posteriormente, volver a evaluar en la misma muestra la velocidad del ultrasonido. De esta manera, se pueden comparar los valores antes y después del procedimiento experimental, para así observar si este modifica de manera significativa la velocidad del ultrasonido.

La cantidad de substancia preservante que una pieza de madera puede retener depende de su capacidad de absorción de fluidos. Debido a la variabilidad en su estructura anatómica, cada especie tiene un comportamiento distinto de acuerdo con el tratamiento aplicado [14]. En términos cuantitativos, cabe mencionar que la retención de sales de boro es función también de la concentración de la sustancia preservante empleada en el tratamiento [15].

La experimentación con sales de boro en la madera se ha orientado a determinar el grado de retención de las mismas. La estrategia se concentra en el estudio de métodos experimentales, y la eficiencia que pueden tener para introducir substancias preservantes en la madera. En ese sentido, [16] consiguieron en probetas de Gmelina arbórea retenciones de 0.32 a $1.28 \mathrm{~kg} \mathrm{~m}^{-3}$, con un método de inmersión-difusión. Así mismo, [17] empleando un método de presión en piezas de madera de Eucalyptus grandis, obtuvieron retenciones de 3.7 a $11.6 \mathrm{~kg} \mathrm{~m}^{-3}$. Finalmente, por medio de un proceso al vacío, [15] reportan retenciones de $17.42 \mathrm{~kg} \mathrm{~m}^{-3}$ para madera de Fagus orientalis y de $15.98 \mathrm{~kg} \mathrm{~m}^{-3}$ para Pinus sylvestris.

Por su parte, empleando un tratamiento de preservación específicamente diseñado, [6] encontraron retenciones de 2.88 a $9.16 \mathrm{~kg} \mathrm{~m}^{-3}$ en madera de Fagus sylvatica, y de 3.25 a $9.57 \mathrm{~kg} \mathrm{~m}^{-3}$ para Pinus sylvestris. Aplicando un tratamiento de preservación con temperatura de $103^{\circ} \mathrm{C},[18]$ lograron una retención de $4.2 \mathrm{~kg} \mathrm{~m}^{-3}$ para madera de Pinus sylvestris, y de $5.7 \mathrm{~kg} \mathrm{~m}^{-3}$ para la de Fagus sylvatica. En cada investigación se emplearon especies de madera, concentraciones de sales de boro y métodos de aplicación diferentes. Por tal razón, los resultados son difíciles de comparar.

Respecto de la aptitud para la preservación de maderas mexicanas, [14] reportan trabajos en los cuales el tratamiento de preservación denominado como "baño caliente-frío" ha sido empleado con éxito. Esta práctica solo necesita de instrumentos accesibles y resulta una técnica económica. En la literatura mexicana relacionada con el tema se encuentra información 
sobre las características de las especies en estudio [19]. Igualmente, existe información relevante sobre los métodos de carácter no destructivo [20].

En ese contexto, en [21] se detalla información sobre la experimentación con sales de boro en la madera y sus estrategias de aplicación. La influencia del tratamiento de impregnación con sales de boro y su efecto en el módulo de elasticidad determinado con ondas de esfuerzo se reporta en [22]. La presente investigación usa como referencia las especies de madera reportadas por estos autores, pero con la diferencia en cuanto al uso de un enfoque y una técnica de evaluación diferentes. Sin embargo, no se encontraron trabajos publicados que integren pruebas de ultrasonido con el efecto de las sales de boro sobre la densidad, la velocidad de onda y el módulo de elasticidad dinámico de la madera.

El objetivo de esta investigación fue observar el efecto del tratamiento de preservación con sales de boro sobre la densidad, la velocidad de onda y el módulo de elasticidad dinámico en las especies de madera G. ulmifolia, S. campanulata y A. religiosa.

\section{Materiales y métodos}

\subsection{Materiales}

Se recolectaron trozas de $0.6 \mathrm{~m}$ de largo, provenientes de las especies Guazuma ulmifolia Lam., Spathodea campanulata P. Beauv. y Abies religiosa (Kunth) Schltdl. \& Cham. en aserraderos del Estado de Michoacán, México $\left(19^{\circ} 10^{\prime} 07^{\prime \prime N}\right.$, $101^{\circ} 53^{\prime} 59^{\prime \prime} \mathrm{O}$ ). Las trozas se aserraron en secciones de $0.2 \mathrm{~m} \mathrm{de}$ ancho. Para cada una de las especies se prepararon lotes de 35 probetas con dimensiones de $0.02 \mathrm{~m} \times 0.02 \mathrm{~m} \times 0.32 \mathrm{~m}$, respectivamente en las direcciones radial, tangencial y longitudinal del plano leñoso y de acuerdo con la norma ISO 3129: 2012 [10]. Las probetas se almacenaron durante 24 meses en una cámara de acondicionamiento con una temperatura de $20^{\circ} \mathrm{C}\left( \pm 1^{\circ} \mathrm{C}\right)$ y una humedad relativa del aire de $65 \%( \pm 2 \%)$, hasta que el peso de la madera fue constante. Las probetas contenían solamente madera de duramen. La madera no presentó anomalías estructurales como fisuras y defectos de crecimiento, como son la desviación de la fibra, los nudos y las bolsas de resinas o ceras. Con el objeto de evitar el flujo excesivo de líquidos en los extremos de las probetas, sus caras laterales fueron cubiertas con pintura vinílica.

\subsection{Métodos}

Se dispusieron probetas de G. ulmifolia, S. campanulata y A. religiosa; estas se preservaron con el método baño caliente- frío con una solución concentrada al 3\%. Para cada especie se determinaron la densidad, la velocidad de onda y el módulo de elasticidad dinámico, antes y después del tratamiento. También se calcularon la absorción y la retención de sales. Se realizaron pruebas de diferencia de medias y se calcularon regresiones para las variables, antes y después de tratamiento.

Se prepararon 30 litros de solución de sales de boro con una concentración al 3\%. La solución consistió en ácido bórico (39.4\%) y borato de sodio (60.6\%), de acuerdo con la norma NMX-C-410-ONNCCE-1999 [23].

Las probetas se impregnaron con el método "baño calientefrío" siguiendo el protocolo propuesto por [14]. La madera se sumergió durante 8 horas en un baño de agua con temperatura de $60^{\circ} \mathrm{C}$. Posteriormente, dichas probetas fueron sumergidas durante 16 horas en un baño frío con la solución de sales de boro, a una temperatura de $23^{\circ} \mathrm{C}$.

Los pesos y dimensiones de las probetas fueron medidos antes y después del tratamiento. Posteriormente, se determinaron la absorción y la retención de las sales para cada probeta.

La absorción se determinó con la fórmula (1) [14]:

$$
A=\frac{P_{2}-P_{1}}{V}
$$

Donde:

$A$ : absorción $\left(\mathrm{kg} \mathrm{m}^{-3}\right)$

$P_{1}$ : peso inicial de la probeta antes del tratamiento $(\mathrm{kg})$

$P_{2}$ : peso final de la probeta después del tratamiento $(\mathrm{kg})$

$V$ : volumen de la probeta después del tratamiento $\left(\mathrm{m}^{3}\right)$

La retención se determinó con la fórmula (2) [15]:

$$
R=\frac{A C}{100}
$$

Donde:

$R$ : retención $\left(\mathrm{kg} \mathrm{m}^{-3}\right)$

$A$ : absorción $\left(\mathrm{kg} \mathrm{m}^{-3}\right)$

$C$ : concentración de la sustancia preservante (\%)

El contenido de humedad en las probetas se determinó con un grupo complementario de otras probetas, con dimensiones de $0.02 \mathrm{~m} \times 0.02 \mathrm{~m} \times 0.06 \mathrm{~m}$. Se calculó la relación del peso de la probeta al momento del ensayo y el peso de esta en estado seco, adaptando la norma ISO 13061-1:2014 [24]. La densidad de la madera se calculó por medio de la relación entre el peso y el volumen en el momento del ensayo, adaptando la norma ISO 13061-2:2014 [25]. 


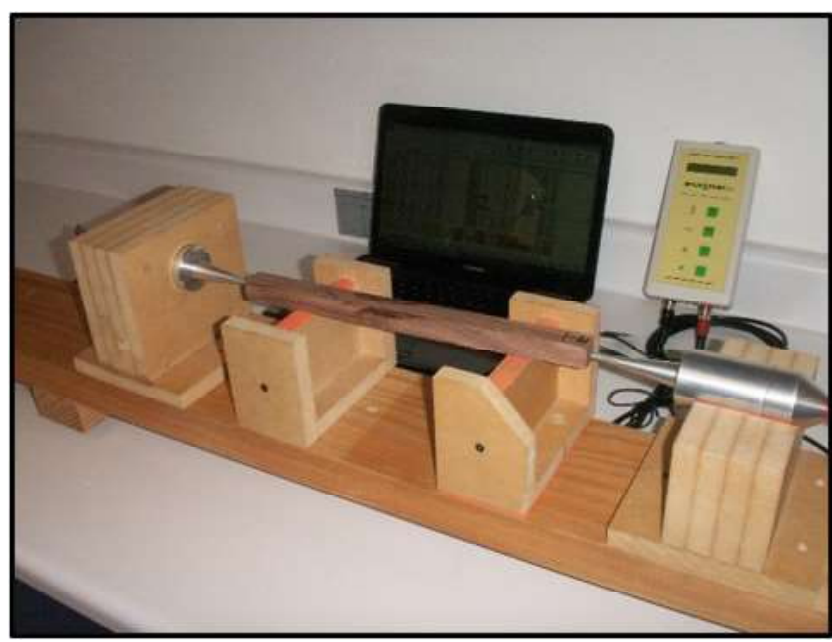

Fig. 1. Dispositivo experimental y aparato Sylvatest $₫$, empleados para las pruebas de ultrasonido.

Una vez terminado el tratamiento de baño caliente-frío, y realizadas las mediciones para calcular la absorción y la retención, las probetas se almacenaron por tres meses en las mismas condiciones en que se estabilizó la madera antes del procedimiento; es decir, en una cámara de acondicionamiento con una temperatura de $20^{\circ} \mathrm{C}\left( \pm 1^{\circ} \mathrm{C}\right)$ y una humedad relativa del aire de $65 \%( \pm 2 \%)$, hasta que alcanzaron un peso constante. La determinación del contenido de humedad y de la densidad, así como las pruebas de ultrasonido, se realizaron antes y después del tratamiento de preservación.

Las pruebas de ultrasonido consistieron en suministrar un impulso ultrasónico de transmisión directa en la dirección longitudinal de la probeta. La velocidad del ultrasonido se calculó con el cociente entre la longitud de las probetas y el tiempo de transmisión medido con el aparato Sylvatest, el cual fue posicionado en un dispositivo para pruebas no destructivas diseñado específicamente para el experimento (veáse Fig. 1). Los parámetros definidos a continuación tienen el subíndice "us", para representar que son parámetros derivados de pruebas de ultrasonido.

El módulo de elasticidad dinámico se calculó con la siguiente fórmula (3) [26]:

$$
E_{u s}=v_{u s} \rho_{C H}
$$

Donde:

$E_{u s}:$ módulo de elasticidad dinámico $\left(\mathrm{N} \mathrm{m}^{-2}\right)$

$v_{u s}:$ velocidad de onda $\left(\mathrm{m} \mathrm{s}^{-1}\right)$

$\rho_{C H}$ : densidad de la madera a un contenido de humedad $\mathrm{CH}\left(\mathrm{kg} \mathrm{m}^{-3}\right)$

\subsection{Diseño experimental}

La unidad experimental consistió en 105 especímenes agrupados en tres muestras de 35 probetas cada una correspondientes a las tres especies en estudio. Las variables de respuesta fueron densidad, velocidad de onda y módulo de elasticidad dinámico, para antes y después de tratamiento. El contenido de humedad de la madera se considera un factor fijo. Se calcularon la media, la desviación estándar y el coeficiente de variación. Para cada muestra se evaluaron el apuntamiento estandarizado y el sesgo estandarizado. Al momento de verificar que los datos provenían de distribuciones normales, se diseñaron dos experimentos siguiendo las recomendaciones de [27].

En el primero, se efectuaron pruebas de diferencia de medias para un nivel de confianza del $95 \%$. El tratamiento de preservación con sales de boro se considera el factor de variación.

La hipótesis nula $\mathrm{H}_{0}: \mathbf{x}_{1}-\mathbf{x}_{2}=0$ se contrastó con la hipótesis alterna $\mathrm{H}_{\mathrm{A}}: \mathbf{x}_{1}-\mathbf{x}_{2} \neq 0$. Los subíndices 1 y 2 representan las variables de respuesta como muestras pareadas, para antes y después del tratamiento.

Las variables de absorción y retención se consideraron variables de respuesta solo después del tratamiento de preservación. Por medio de las mismas, se preparó un segundo experimento de análisis de varianza para un nivel de confianza del $95 \%$. En este caso el factor de variación fue la especie. La hipótesis nula $\mathrm{H}_{0}: \mathbf{x}_{1}-\mathbf{x}_{2}=0$ se contrastó con la hipótesis alternativa $\mathrm{H}_{\mathrm{A}}: \mathbf{x}_{1}-\mathbf{x}_{2} \neq 0$. Los subíndices 1 y 2 representan las variables de respuesta para cada una de las especies.

Adicionalmente, después del tratamiento se calcularon modelos de regresión lineales y sus coeficientes de determinación entre las variables de densidad, velocidad de onda y módulo de elasticidad dinámico; todo esto en función de las mismas variables antes del tratamiento.

\section{Resultados y discusión}

La Tabla 1 presenta los resultados de la densidad, de la velocidad de onda y del módulo de elasticidad dinámico para las especies G. ulmifolia, S. campanulata y A. religiosa. Los datos están tabulados para antes y después del tratamiento de preservación. La retención y absorción de sales de boro se tabularon solo para después del tratamiento.

La magnitud del contenido de humedad, antes y después del tratamiento, fue en promedio de $11 \%$, varió $2.2 \%$ y sus coeficientes de variación se diferenciaron $1.2 \%$. De forma que se consideró este parámetro como no influyente en los resultados. 
Las pruebas de normalidad para todas las variables de respuesta (véase Tabla 2) confirmaron que los datos de las muestras provinieron de distribuciones normales.

Las pruebas de diferencia de medias entre los resultados, antes y después del tratamiento (véase Tabla 2), indicaron que para la densidad de la madera se encontró una diferencia estadísticamente significativa para $S$. campanulata y A. religiosa. En contraste, la densidad no cambió para G. ulmifolia. La velocidad de onda de A. religiosa fue la que únicamente denotó una diferencia estadísticamente significativa. Dicha característica no se modificó para G. ulmifolia y S. campanulata. En cuanto al módulo de elasticidad dinámico los valores para G. ulmifolia y A. religiosa fueron estadísticamente diferentes, antes y después de tratamiento. En cambio, el módulo de elasticidad dinámico no se alteró para $S$. campanulata.

Los análisis de varianza para la absorción y la retención (véase Tabla 3) proporcionaron diferencias estadísticamente significativas entre las tres especies. Es decir, cada una de las tres maderas absorbió y retuvo sales de boro en una cantidad diferente.

Los coeficientes de determinación de las regresiones entre las variables densidad de la madera, velocidad de onda y módulo de elasticidad dinámico (véase Tabla 4) verificaron las corres- pondencias entre los resultados para las especies G. ulmifolia y $S$. campanulata, antes y después del tratamiento. Un resultado particular fue el de la especie $A$. religiosa, donde la velocidad de onda y el módulo de elasticidad dinámico mostraron coeficientes de determinación muy bajos.

Estos resultados se muestran gráficamente en la Fig. 2, antes y después del tratamiento, donde se aprecian las dispersiones de los resultados de cada variable para las tres especies estudiadas, extraídos de las 35 probetas estudiadas. En efecto, los datos de la velocidad de onda y del módulo de elasticidad dinámico son correspondientes entre sí para las tres especies, lo cual se tradujo en coeficientes de determinación altos (véase Tabla 4). En cambio, para A. religiosa los coeficientes de determinación de las regresiones fueron particularmente débiles, en cuanto a la velocidad de transmisión de onda y el módulo de elasticidad dinámico. Así mismo, la dispersión de sus resultados no mostró una dependencia.

Después del tratamiento la densidad de G. ulmifolia aumentó en un $2.6 \%$ y la de $A$. religiosa en un $3.6 \%$. En contraste, la densidad de $\mathrm{S}$. campanulata disminuyó en un $5.6 \%$ (véase Tabla 1). La velocidad del ultrasonido en G. ulmifolia aumentó en un $1.8 \%$; la de $S$. campanulata aumentó en un $2.5 \%$; y la velocidad del ultrasonido en $A$. religiosa aumentó en un $6.4 \%$. Esta combinación de resultados repercutió en que el

Tabla 1. Densidad de la madera, velocidad de onda, módulo de elasticidad dinámico, absorción y retención de sales de boro.

\begin{tabular}{|c|c|c|c|c|c|c|c|c|}
\hline & $\begin{array}{l}\rho_{C H} \\
\mathrm{~kg} \mathrm{~m}^{-3} \\
\end{array}$ & $\begin{array}{l}v_{u s} \\
\mathrm{~m} \mathrm{~s}^{-1}\end{array}$ & $\begin{array}{l}E_{u s} \\
\mathrm{MN} \mathrm{m}{ }^{-2}\end{array}$ & $\begin{array}{l}\rho_{C H} \\
\mathrm{~kg} \mathrm{~m}^{-3}\end{array}$ & $\begin{array}{l}v_{u s} \\
\mathrm{~m} \mathrm{~s}^{-1}\end{array}$ & $\begin{array}{l}E_{u s} \\
\mathrm{MN} \mathrm{m}^{-2}\end{array}$ & $\begin{array}{l}A \\
\mathrm{~kg} \mathrm{~m}^{-3}\end{array}$ & $\begin{array}{l}R \\
\mathrm{~kg} \mathrm{~m}^{-3}\end{array}$ \\
\hline & \multicolumn{3}{|c|}{ Antes del tratamiento } & \multicolumn{5}{|c|}{ Después del tratamiento } \\
\hline & \multicolumn{3}{|c|}{ G. ulmifolia } & \multicolumn{5}{|c|}{ G. ulmifolia } \\
\hline $\mathbf{x}$ & 730 & 3952 & 11389 & 749 & 4022 & 12114 & 323.2 & 9.7 \\
\hline$\sigma$ & 46.50 & 288 & 1208 & 42.06 & 287 & 1304 & 13.5 & 0.4 \\
\hline \multirow[t]{2}{*}{$C V$} & 6.4 & 7.3 & 10.6 & 5.6 & 7.1 & 10.8 & 4.2 & 4.2 \\
\hline & \multicolumn{3}{|c|}{ S. campanulata } & \multicolumn{5}{|c|}{ S. campanulata } \\
\hline $\mathbf{x}$ & 357 & 3693 & 4860 & 336 & 3787 & 4819 & 477.0 & 14.3 \\
\hline$\sigma$ & 30.40 & 382 & 776 & 25.62 & 384 & 793 & 20.0 & 0.6 \\
\hline \multirow[t]{2}{*}{$C V$} & 8.5 & 10.3 & 16.0 & 7.6 & 10.1 & 16.5 & 4.2 & 4.2 \\
\hline & \multicolumn{3}{|c|}{ A. religiosa } & \multicolumn{5}{|c|}{ A. religiosa } \\
\hline $\mathbf{x}$ & 419 & 5944 & 14888 & 434 & 6354 & 17557 & 240.9 & 7.2 \\
\hline$\sigma$ & 28.67 & 399 & 2251 & 27.93 & 177 & 1869 & 42.0 & 1.3 \\
\hline$C V$ & 6.8 & 6.7 & 15.1 & 6.4 & 2.8 & 10.6 & 17.4 & 17.4 \\
\hline
\end{tabular}


Tabla 2. Pruebas de normalidad y de diferencias de medias.

\begin{tabular}{|c|c|c|c|c|c|c|}
\hline \multirow[b]{2}{*}{ Prueba } & \multicolumn{2}{|c|}{$\begin{array}{l}\text { G. ulmifolia } \\
\text { Normalidad }\end{array}$} & \multicolumn{2}{|c|}{ S. campanulata } & \multicolumn{2}{|c|}{ A. religiosa } \\
\hline & $\mathrm{AT}$ & $\mathrm{DT}$ & $\mathrm{AT}$ & $\mathrm{DT}$ & $\mathrm{AT}$ & $\mathrm{DT}$ \\
\hline & $\rho_{C H}$ & & $\rho_{C H}$ & & $\rho_{C H}$ & \\
\hline Sesgo & 0.3418 & 0.4859 & 0.7589 & 0.9192 & 0.9258 & 0.6457 \\
\hline \multirow[t]{2}{*}{ Apuntamiento } & -1.4128 & -1.0136 & -0.7476 & -0.5998 & -0.7036 & -0.5153 \\
\hline & $v_{u s}$ & & $v_{u s}$ & & $v_{u s}$ & \\
\hline Sesgo & 0.0133 & -0.4991 & 0.1281 & 0.3183 & -1.6418 & 0.631 \\
\hline \multirow[t]{2}{*}{ Apuntamiento } & -1.2488 & -1.2967 & -0.8634 & -0.8749 & -0.7011 & -0.8399 \\
\hline & $E_{u s}$ & & $E_{u s}$ & & $E_{u s}$ & \\
\hline Sesgo & -1.5547 & -1.7925 & 0.2873 & 0.4932 & 0.3649 & 1.0757 \\
\hline \multirow[t]{3}{*}{ Apuntamiento } & -0.2144 & -0.5797 & -0.4342 & -0.6435 & 0.2155 & -0.6607 \\
\hline & \multicolumn{6}{|c|}{ Diferencia de medias } \\
\hline & \multirow{2}{*}{\multicolumn{2}{|c|}{$\begin{array}{l}\rho_{C H} \\
\rho_{C H} \text { AT vs. } \rho_{C H} \text { DT } \\
0.0748\end{array}$}} & \multicolumn{2}{|l|}{$\rho_{C H}$} & \multicolumn{2}{|l|}{$\rho_{C H}$} \\
\hline Valor $P_{(\alpha=0.05)}$ & & & \multicolumn{2}{|c|}{$\begin{array}{l}\rho_{C H} \text { AT vs. } \rho_{C H} \text { DT } \\
0.0024^{*}\end{array}$} & \multicolumn{2}{|c|}{$\begin{array}{l}\rho_{C H} \text { AT vs. } \rho_{C H} \text { DT } \\
0.0399 *\end{array}$} \\
\hline Valor $\mathrm{P}_{(\alpha=0.05)}$ & \multicolumn{2}{|c|}{$\begin{array}{l}v_{u s} \text { AT vs. } v_{u s} \text { DT } \\
0.3128\end{array}$} & \multicolumn{2}{|c|}{$\begin{array}{l}v_{u s} \mathrm{AT} \text { vs. } v_{u s} \mathrm{DT} \\
0.3056\end{array}$} & \multicolumn{2}{|c|}{$\begin{array}{l}v_{u s} \mathrm{AT} \mathrm{vs.} v_{u s} \mathrm{DT} \\
<0.001^{*}\end{array}$} \\
\hline Valor $P_{(\alpha=0.05)}$ & \multicolumn{2}{|c|}{$\begin{array}{l}E_{u s} \text { AT vs. } E_{u s} \text { DT } \\
0.0185^{*}\end{array}$} & \multicolumn{2}{|c|}{$\begin{array}{l}E_{u s} \text { AT vs. } E_{u s} \text { DT } \\
0.8255\end{array}$} & \multicolumn{2}{|c|}{$\begin{array}{l}E_{u s} \text { AT vs. } E_{u s} \mathrm{DT} \\
<0.001 *\end{array}$} \\
\hline
\end{tabular}

$\rho_{C H}=$ densidad; $v_{u s}=$ velocidad de onda; $E_{u s}=$ módulo de elasticidad dinámico; $A T=$ antes tratamiento; $D T=$ después tratamiento; $\mathrm{P}_{(\alpha=0.05)}<0.05$ : diferencia significativa para un nivel del $95 \%$ de confianza.

Tabla 3. Análisis de varianza.

\begin{tabular}{|c|c|c|c|c|c|}
\hline Fuente & Suma de cuadrados & GL & Cuadrado medio & Razón-F & $P_{(\alpha=0.05)}$ \\
\hline & Absorción & & & & \\
\hline Entre grupos & 1000000 & 2 & 502435 & 643.1 & $<0.0001^{*}$ \\
\hline Intra grupos & 79691 & 102 & 781.3 & - & - \\
\hline \multirow[t]{2}{*}{ Total } & 1080000 & 104 & - & - & - \\
\hline & Retención & & & & \\
\hline Entre grupos & 905 & 2 & 452.3 & 643.7 & $<0.0001^{*}$ \\
\hline Intra grupos & 71.7 & 102 & 0.7026 & - & - \\
\hline Total & 976.2 & 104 & - & - & - \\
\hline
\end{tabular}


Tabla 4. Regresiones.

\begin{tabular}{|c|c|c|c|}
\hline$y \mathrm{DT}=a x \mathrm{AT}+b$ & $a$ & $b$ & $R^{2}$ \\
\hline & \multicolumn{3}{|l|}{ G. ulmifolia } \\
\hline$\rho_{C H}$ DT $=f\left(\rho_{C H}\right.$ AT $)$ & 0.872 & 112.8 & 0.93 \\
\hline$v_{u s} \mathrm{DT}=\mathrm{f}\left(v_{u s} \mathrm{AT}\right)$ & 0.957 & 240 & 0.93 \\
\hline \multirow[t]{2}{*}{$E_{u s} \mathrm{DT}=\mathrm{f}\left(E_{u s} \mathrm{AT}\right)$} & 0.996 & 775 & 0.85 \\
\hline & \multicolumn{3}{|l|}{ S. campanulata } \\
\hline$\rho_{C H} \mathrm{DT}=\mathrm{f}\left(\rho_{C H} \mathrm{AT}\right)$ & 0.813 & 45.9 & 0.92 \\
\hline$v_{u s} \mathrm{DT}=\mathrm{f}\left(v_{u s} \mathrm{AT}\right)$ & 0.985 & 150 & 0.97 \\
\hline \multirow[t]{2}{*}{$E_{u s} \mathrm{DT}=\mathrm{f}\left(E_{u s} \mathrm{AT}\right)$} & 0.974 & 95 & 0.91 \\
\hline & \multicolumn{3}{|l|}{ A. religiosa } \\
\hline$\rho_{C H} \mathrm{DT}=\mathrm{f}\left(\rho_{C H} \mathrm{AT}\right)$ & 0.959 & 31.5 & 0.97 \\
\hline$v_{u s} \mathrm{DT}=\mathrm{f}\left(v_{u s} \mathrm{AT}\right)$ & 0.073 & 5923 & 0.03 \\
\hline$E_{u s} \mathrm{DT}=\mathrm{f}\left(E_{u s} \mathrm{AT}\right)$ & 0.432 & 11121 & 0.27 \\
\hline
\end{tabular}

$\rho_{C H}=$ densidad; $v_{u s}=$ velocidad de onda; $E_{u s}=$ módulo de elasticidad dinámico;

$A T=$ antes tratamiento; $D T=$ después tratamiento; $R^{2}=$ coeficiente de determinación.
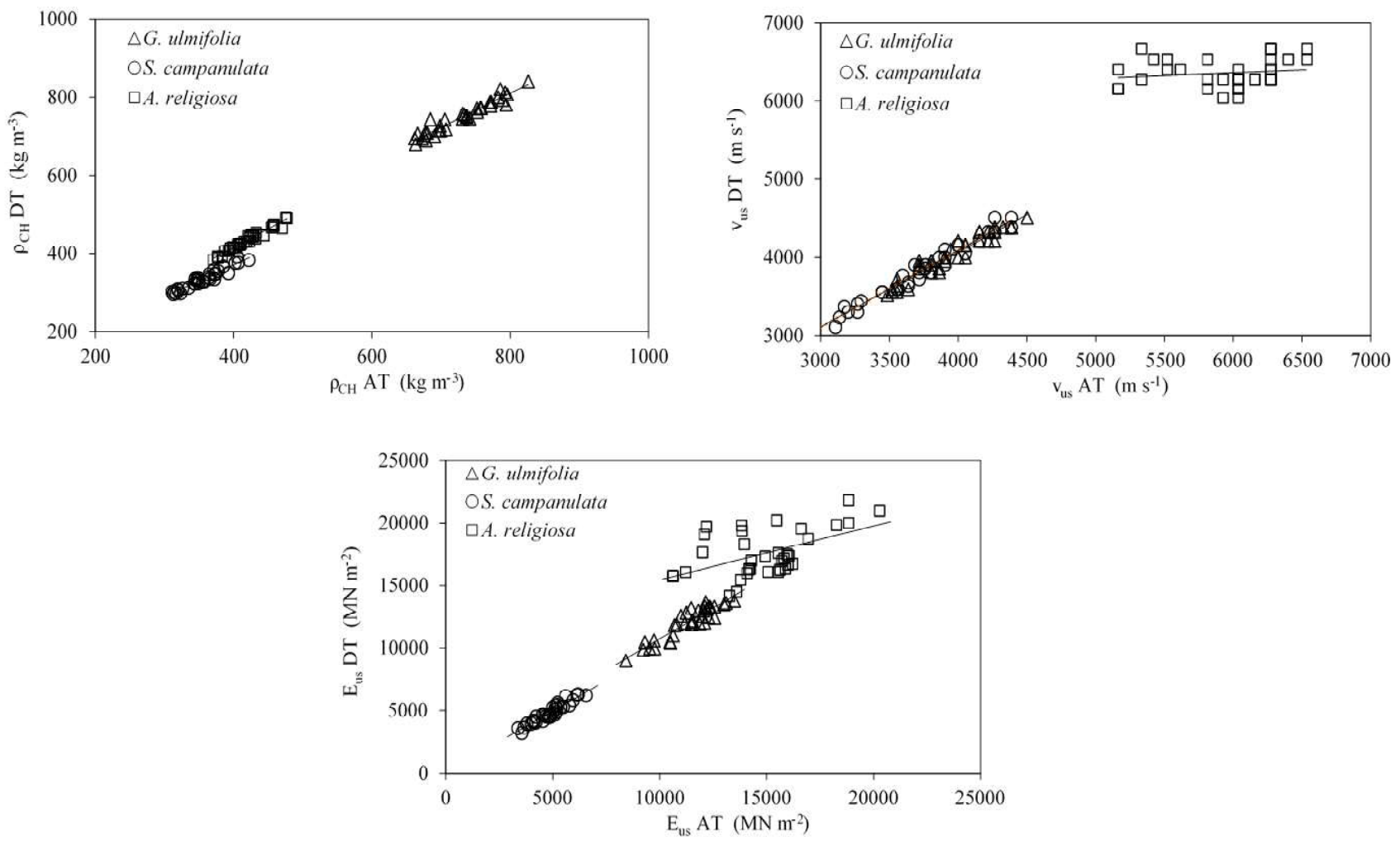

Fig. 2. Dispersiones de la densidad, de la velocidad de onda y del módulo de elasticidad dinámico. 
módulo de elasticidad dinámico de G. ulmifolia aumentó en un $6.4 \%$, y el de $A$. religiosa en un $17.9 \%$. Por otro lado, el módulo de elasticidad dinámico de $S$. campanulata disminuyó en un $1 \%$. Bajo ese mismo contexto, no se observa una discrepancia importante entre las especies estudiadas, a pesar de que los coeficientes de variación son diferentes para antes y después del tratamiento.

La variación de la densidad ocasionada por el tratamiento de preservación puede ser explicada, entre otros factores, por los cambios sufridos en la estructura anatómica de la madera. El espesor de la pared celular de la madera, así como su capacidad de retención de las sales, que son características propias a cada especie, pueden incrementar la densidad de la madera. Un caso particular es el de la madera de $S$. campanulata, la cual retuvo más sales de boro donde, sin embargo, mostró una disminución en su densidad.

La absorción, que es un parámetro intermedio necesario para calcular la retención, presenta coeficientes de variación iguales a los de la retención (véase Tabla 1). Las especies G. ulmifolia y $A$. religiosa tienen retenciones similares. En contraste, $S$. campanulata retiene más sales de boro: $47.5 \%$ en comparación con G. ulmifolia, y $98 \%$ en referencia con $A$. religiosa.

Los valores promedio de retención de sales de boro para las tres especies (véase Tabla 1) fueron mayores que el límite inferior tóxico de retención reportado en trabajos anteriores. De acuerdo con [28], el umbral mínimo de retención, necesario para proteger a la madera de las pudriciones ocasionadas por hongos, está comprendido en el intervalo que va de 0.2 a $4.7 \mathrm{~kg} \mathrm{~m}^{-3}$. Por su parte, [29] propone una retención mínima de $1 \mathrm{~kg} \mathrm{~m}^{-3}$ para protección de la madera en condiciones de uso en exteriores. La Asociación Americana de Preservadores de Madera [30] recomienda una retención mínima de sales de boro de $2.72 \mathrm{~kg} \mathrm{~m}^{-3}$ en la madera, esto para elementos estructurales empleados en interiores.

En términos cuantitativos, los resultados de retención son comparables con los de [31] para un tratamiento por inmersión. Este autor presenta valores de retención de sales de boro para nueve especies de madera tropicales: Hymenaea courbaril $23.2 \mathrm{~kg} \mathrm{~m}^{-3}$, Terminalia amazonia $12.9 \mathrm{~kg} \mathrm{~m}^{-3}$, Hieronyma alchorneoides $8.1 \mathrm{~kg} \mathrm{~m}^{-3}$, Cedrela odorata $5.7 \mathrm{~kg} \mathrm{~m}^{-3}$, Tectona grandis $9.1 \mathrm{~kg} \mathrm{~m}^{-3}$, Cupressus lusitanica $14.9 \mathrm{~kg} \mathrm{~m}^{-3}$, Enterolobium cyclocarpum $7 \mathrm{~kg} \mathrm{~m}^{-3}$, Calophyllum brasiliense $15 \mathrm{~kg} \mathrm{~m}^{-3}$ y Gmelina arborea $8.7 \mathrm{~kg} \mathrm{~m}^{-3}$. Los valores de esta investigación para $C$. odorata pueden considerarse similares a los resultados presentados por [31]. De aquí se desprende que las especies estudiadas pueden ser tratadas con sales de boro, y de esa manera satisfacer los estándares necesarios para su empleo en condiciones donde se requiere un método de protección.
Los argumentos presentados confirman el paradigma que rige en investigación e ingeniería de la madera: es necesario caracterizar los comportamientos físico-mecánicos de la madera con un enfoque de experimentación, caso por caso y para una especie en particular. Cada procedimiento debe estar relacionado con las variables de referencia de las condiciones de ensayo, por ejemplo, la densidad y el contenido de humedad de la madera. También debe haber relación con los datos derivados del tamaño de una muestra representativa, observada estadísticamente. Una vez que se obtienen datos integrantes y estadísticamente representativos, se pueden proponer tendencias en el comportamiento mecánico general para una especie en específico, o por agrupamiento de varias de ellas que denoten un comportamiento similar [22].

\section{Conclusiones}

Las maderas de G. ulmifolia, S. campanulata y A. religiosa pueden ser impregnadas con sales de boro con el método baño caliente-frío, sin reducir su densidad, velocidad de onda y módulo dinámico. Cada especie se comporta de manera diferente. Para cada una de las especies estudiadas, los valores promedio de retención de sales de boro son mayores que el límite inferior tóxico de retención reportado en trabajos anteriores. Cuantitativamente, los resultados son comparables con los reportados en la bibliografía.

Para la densidad de la madera se encontró una diferencia estadísticamente significativa para $S$. campanulata y $A$. religiosa. En contraste, la densidad no cambió para G. ulmifolia. La velocidad de onda de $A$. religiosa fue la que únicamente denotó una diferencia estadísticamente significativa. Dicha característica no se modificó para G. ulmifolia y $S$. campanulata. El módulo de elasticidad dinámico los valores para G. ulmifolia, $S$. campanulata y $A$. religiosa fueron estadísticamente diferentes, antes y después de tratamiento.

A manera de prospectiva, los resultados de la investigación sugieren el empleo del método baño-caliente frío para impregnar madera con sales de boro. Para su aplicación a nivel industrial se recomienda realizar estudios con piezas de madera con dimensiones de empleo en la industria.

\section{Agradecimientos}

A los alumnos de la Facultad de Ingeniería en Tecnología de la Madera, de la Universidad Michoacana de San Nicolás de Hidalgo (UMSNH), por su colaboración en los trabajos de laboratorio. La investigación estuvo patrocinada por la Coordinación de la Investigación Científica de la UMSNH. 


\section{Referencias}

[1] R. W. Meyer, R. M. Kellogg, Structural Use of Woods in Adverse Environments, New York, U. S. A.: Van Nostrand Reinhold, 1982.

[2] R. A. Zabel, J. J. Morell, Wood Microbiology: Decay and Its Prevention, Cambridge, U. K.: Academic Press, 1992.

[3] R. M. Rowell, R. J. Barbour, Archeological Wood Properties, Chemistry, and Preservation, Washington, D C, USA:American Chemical Society, 1990.

[4] L. Helsen, E. Van den Bulck, "Review of disposal technologies for chromated copper arsenate (CCA) treated wood waste, with detailed analyses of thermochemical conversion processes," Environmental Pollution, vol. 134, no. 2, pp. 301-314, 2005.

[5] A. Temiz, G. Alfredsen, M. Eikenes, N. Terzev, "Decay resistance of wood treated with boric acid and tall oil derivates," Bioresource Technology, vol. 99, no. 7, pp. 2102-2106, 2008.

[6] G. Tondi, S. Wieland, N. Lemenager, A. Petutschnigg, A. Pizzi, M. F. Thevenon, "Efficacy of tannin in fixing boron in wood: fungal and termite resistance," BioResources, vol. 7, no. 1, pp. 1238-1252, 2012.

[7] Tsunoda, K. "Preservatives properties of vapor-borontreated wood and wood based composites," Journal of Wood Science, vol. 47, no. 2, pp. 149-53, 2001.

[8] D. D. Obanda, T. F. Shupe, H. M. Barnes, "Reducing leaching of boron-based wood preservatives. A review of research," Bioresource Technology, vol. 99, no. 15, pp. 7312-7322, 2008.

[9] D.E. Breyer, K. J. Fridley, D. G. Pollock, K. E. Cobeen, Design of Wood Structures ASD. Columbus, U. S. A.: McGraw-Hill, 2003.

[10] International Organization for Standardization, ISO 3129:2012. Wood. Sampling methods and general requirements for physical and mechanical testing of small clear wood specimens, Geneva, Switzerland: International Organization for Standardization, 2012.

[11] Asociación Española de Normalización y Certificación, UNE-EN 384:2010. Madera Estructural. Determinación de los valores característicos de las propiedades mecánicas y la densidad. Madrid, España: Asociación Española de Normalización y Certificación, 2010.

[12] C. H. S. Del Menezzi, M. R. S. Amorim, M. A. Costa, L. R. O, Garcez, "Evaluation of Thermally Modified Wood by Means of Stress Wave and Ultrasound Nondestructive Methods," Materials Science (Medziagotyra), vol. 20, no. 1,pp. 61-66, 2014.

[13] R. A. Gonçalves, J. Trinca, B. P. Pellis, "Elastic constants of wood determined by ultrasound using three geometries of specimens," Wood Science and Technology, vol. 48, no. 2, pp. 269-287, 2014.

[14] L. E. A. Ávila Calderón, M. A. Herrera Ferreyra, D. Raya González, Preservación de la Madera en México, Morelia, Universidad Michoacana de San Nicolás de Hidalgo, 2012.

[15] H. Simsek, E. Baysal, H. Peker, "Some mechanical properties and decay resistance of wood impregnated with environmentally friendly borates," Construction and Building Materials, vol. 24, no. 11,pp. 2279-2284, 2010.

[16] A. Berrocal, F. Muñoz, G. González, "Ensayo de penetrabilidad de dos preservantes a base de boro en madera de melina (Gmelina arborea) crecida en Costa Rica," Kurú: Revista Forestal, vol. 1, no. 3, pp. 1-12, 2004.

[17] T. K. Dhamodaran, R. Gnanaharan, "Boron impregnation treatment of Eucalyptus grandis Wood," Bioresource Technology, vol. 98, no. 11, pp. 2240-2242, 2007.

[18] S. Salman, A. Pétrissans, M. F. Thévenon, S. Dumarcay, D. Perrin, B. Pollier, P. Gérardin, "Development of new wood treatments combining boron impregnation and thermo modification: effect of additives on boron leachability," European Journal of Wood Products, vol. 72, no. 3, pp. 355-365, 2014.

[19] J. R. Sotomayor Castellanos, Banco FITECMA de características físico-mecánicas de maderas mexicanas, Morelia, México: Universidad Michoacana de San Nicolás de Hidalgo, 2015.

[20] J. R. Sotomayor Castellanos, Caracterización mecánica de la madera con métodos no destructivos, Morelia, México: Universidad Michoacana de San Nicolás de Hidalgo, 2014.

[21] J. Cruz de León, Manual para la protección contra el deterioro de la madera. Guadalajara, México: Comisión Nacional Forestal, 2010.

[22] J. R. Sotomayor Castellanos, and S. Correa Jurado, "Retención de sales de boro en la madera y su efecto en el módulo de elasticidad dinámico," Científica, vol. 24, no. 1, pp. 1-19, 2016.

[23] Organismo Nacional de Normalización y Certificación de la Construcción y Edificación, Norma Mexicana NMXC-410-ONNCCE-1999. Industria de la Construcción. Vivienda de Madera. Retención y Penetración de Sustancias Preservadoras en Madera. Métodos de Prueba. México, México: Organismo Nacional de Normalización y Certificación de la Construcción y Edificación, 1999.

[24] International Organization for Standardization, ISO 13061-1:2014. Physical and mechanical properties of wood. Test methods for small clear wood specimens. Part 1: Determination of moisture content for physical and mechanical tests, Geneva, Switzerland: International Organization for Standardization, 2014. 
[25] International Organization for Standardization, ISO 13061-2:2014. Physical and mechanical properties of wood. Test methods for small clear wood specimens. Part 2: Determination of density for physical and mechanical tests, Geneva, Switzerland: ISO, 2014.

U. Dackermann, K. Crews, B. Kasal, J. Li, M. Riggio, F.

[26] Rinn, T. Tannert, "In situ assessment of structural timber using stress-wave measurements," Materials and Structures, vol. 47, no. 5, pp. 787-803, 2014.

[27] H. Gutiérrez Pulido, R. de la Vara Salazar, Análisis y diseño de experimentos, México: Mc Graw Hill, 2012.

[28] C. Freitag, J. J. Morrell, "Development of threshold values for boron and fluoride in non-soil contact applications," Forest Products Journal, vol. 55, no. 4, pp. 97-101, 2005.

[29] S. N. Kartal, "Combined effect of boron compounds and heat treatments on wood properties: boron release and decay and termite resistance," Holzforschung, vol. 60, no. 4, pp. 455-458, 2006.

[30] American Wood-Preservers' Association, P5-07 Standard for Waterborne Preservatives, Chicago, U. S. A.: American Wood-Preservers' Association, 2014.

[31] J. D. Alfaro Pérez, "Estudio de retención y penetración de tres preservantes comerciales en nueve especies maderables presentes en Costa Rica," Ingeniería, vol. 23, no.1, pp. 107-119, 2013. 
